\title{
Aberrations of Betanin Sensitized Gelatin Microlenses
}

\author{
D. Vasiljević* , B. Murić, D. Pantelić and B. Panić \\ Institute of Physics, University of Belgrade, Serbia
}

\begin{abstract}
Aberrations of the concave aspherical microlenses are presented. Microlenses are produced by using betanin sensitized gelatin and $532 \mathrm{~nm}$ laser irradiation (2nd Nd:YAG harmonic). Aberrations are analyzed by calculation of the longitudinal spherical aberration and the root mean square RMS spot radius. Three presented microlenses had good image quality for the large $F$ number and the moderate field angle.
\end{abstract}

PACS numbers: 42.15.Fr, 42.70.Gi, 81.05.Zx, 42.79.Bh

\section{Introduction}

Manufacturing and application of microlenses is rapidly expanding field in optics. With each day number of possible applications of microlenses is growing. Some of applications are cell phone cameras, medical devices, optical data storage, wavefront sensing, diffusers, Lunenburg and geodesic lenses, waveguides $[1,4]$. Like classical lenses, microlenses can have a concave or a convex shape. Microlenses usually have an aspherical profile, but can also have a spherical profile [5,6]. Standard dimensions for microlenses are from several tens of micrometers to one millimeter.

In this paper only geometrical aberrations (the longitudinal spherical aberration and the root mean square (RMS) spot radius) of betanin sensitized gelatin (BSG) microlenses are calculated. The diffraction effects are not considered because the geometrical aberrations are several times greater than the diffraction effects. All aberrations are analyzed for moderate field of view $\left(2 \omega=10^{\circ}\right)$.

\section{Production of microlenses}

The first step in the process of microlenses production is the preparation of the BSG film. The BSG film is prepared by the gravity settling method [7] on a microscope glass plate (the procedure outlined in Table I). The betanin is natural, water soluble pigment which can be obtained from red beet roots. In our experiment we used betanin in a form of commercially available food dye (E 162) to sensitize gelatin. The betanin has maximum absorption at $535 \mathrm{~nm}$.

After drying BSG film thickness of $100 \mu \mathrm{m}$ was obtained. Microlenses were formed after illumination by the second harmonic $(532 \mathrm{~nm})$ unfocused Nd:YAG laser beam. For all microlenses laser power was the same $(200 \mathrm{~mW})$ and only exposure time was different (2 s,

\footnotetext{
* corresponding author; e-mail: darko@phy.bg.ac.yu
}

TABLE I

The betanin sensitized gelatin film preparation.

\begin{tabular}{c|l}
\hline \hline Step & Description \\
\hline 1 & $\begin{array}{l}\text { swell } 5 \mathrm{~g} \text { commercial-quality gelatin (edible gelatin) } \\
\text { in } 100 \mathrm{ml} \text { deonized water for one hour }\end{array}$ \\
\hline 2 & $\begin{array}{l}\text { heat the suspension in a thermostated water bath at } \\
50^{\circ} \mathrm{C}, \text { with stirring }\end{array}$ \\
\hline 3 & add $5 \mathrm{~g}$ of betanin with stirring \\
\hline 4 & $\begin{array}{l}\text { centrifuge betanin sensitized gelatin solution in order } \\
\text { to remove impurities }\end{array}$ \\
\hline 5 & $\begin{array}{l}\text { pipette out of } 1 \mathrm{ml} \text { of betanin sensitized gelatin solu- } \\
\text { tion onto a precisely leveled and well-cleaned micro- } \\
\text { scope glass plate }\end{array}$ \\
\hline 6 & $\begin{array}{l}\text { dry the betanin sensitized gelatin film in the dark, } \\
\text { overnight, in relatively stable environmental condi- } \\
\text { tions }\end{array}$
\end{tabular}

$5 \mathrm{~s}, 10 \mathrm{~s}$ ). With different exposure times we obtained microlenses with different effective focal lengths and $F$ numbers. The long term stability of BSG microlenses was obtained without additional chemical processing. Details about the experimental setup and the possible explanation for forming microlenses are given in $[8,9]$. The lens shape is concave with aspherical profile, as verified by stylus profilometry (Talystep ${ }^{\mathrm{TM}}$ surface profiler manufactured by Taylor-Hobson Ltd.). The original profile of microlens is given in Fig. 1.

\section{Geometry of microlenses}

The microlenses are in optical design program represented as standard lenses with two surfaces one flat and the other concave. The concave surface is characterized by even asphere surface. The detailed description is given in [10]. For given microlenses the even asphere surface is defined as: 


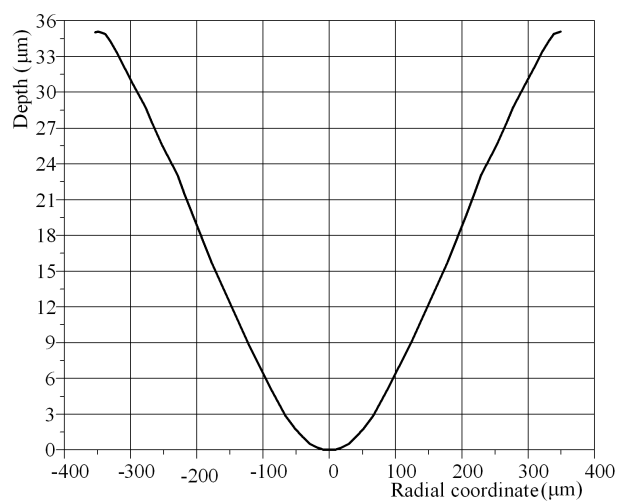

Fig. 1. The profile of microlens.

$$
z=\alpha_{1} r^{2}+\alpha_{2} r^{4},
$$

where $r=\sqrt{x^{2}+y^{2}}$ is the aperture of the lens (the radial coordinate), $\alpha_{1}, \alpha_{2}$ are the polynomial coefficients.

The basic parameters for three microlenses (signed ML1 to ML3) are given in Table II.

TABLE II

Basic parameters for aspherical concave microlenses.

\begin{tabular}{l|c|c|c}
\hline \hline & ML1 & ML2 & ML3 \\
\hline time of laser irradiation [s] & 2 & 5 & 10 \\
effective focal length EFL [mm] & -0.17 & -0.11 & -0.08 \\
$F$ number $(F / \#)[-]$ & 1.8 & 1.3 & 0.8 \\
lens thickness $[\mu \mathrm{m}]$ & 99 & 98 & 98 \\
coefficient $\alpha_{1}[-]$ & -5.4 & -8.1 & -11.5 \\
coefficient $\alpha_{2} \times 10^{-5}[-]$ & 2.13 & 4.24 & 8.17
\end{tabular}

The index of refraction for BSG layer is assumed to be same as index of refraction for gelatin $(n=1.52$ [11] because of small percent of betanin in BSG layer.

\section{Aberrations of microlenses}

Aberrations of the three aspherical concave microlenses are calculated in standard lens design program. For all microlenses moderate field of view $\left(2 \omega=10^{\circ}\right)$ is used. All presented microlenses have rather large $F$ number (from $f / 1.8$ to $f / 0.7)$. From standard theory of aberrations it is well known fact that an optical system with a large $F$ number ought to be complex in order to have small aberrations. For single microlens it cannot be expected to have aberrations smaller than diffraction, so diffraction effects are not considered.

In design of optical systems the most important aberration to correct is the spherical aberration. The longitudinal spherical aberration (LSA) as a function of clear aperture for all three microlenses is presented in Fig. 2. It is customary to represent the clear aperture as a non-dimensional value. In this way spherical aberrations for different optical systems can be compared.

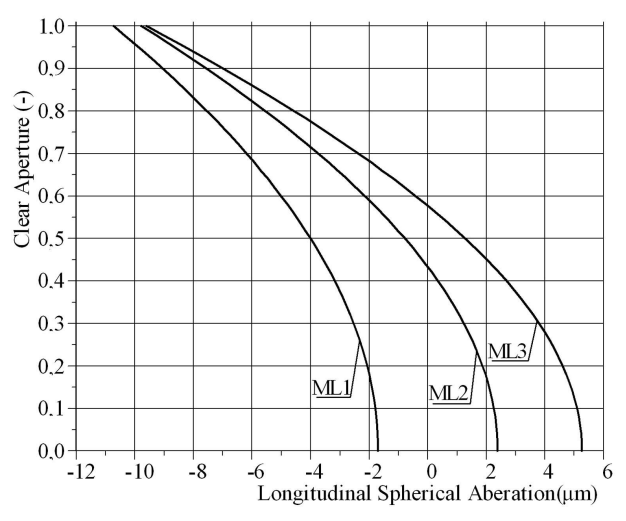

Fig. 2. The LSA as a function of the clear aperture.

The theory of aberrations gives the aberration tolerance for the balanced spherical aberration as [12]:

$$
A_{i}=0.955 \lambda
$$

where $\lambda$ is the wavelength. This relation shows the maximum value of the spherical aberration in order to have diffraction limited optical system. In our case the tolerance for the spherical aberration is $0.5 \mu \mathrm{m}$. As can be seen from Fig. 2, microlenses has similar maximum values for the LSA (from $-9.6 \mu \mathrm{m}$ for ML3 to $-10.7 \mu \mathrm{m}$ for ML1). The calculated values for the LSA are much larger then the aberration tolerance, so microlenses are aberration limited optical systems.

The best way to describe the optical performance of microlenses is through the RMS spot radius. The RMS spot radius is calculated by tracing large number of rays through the optical system. The algorithm for calculation of the RMS spot radius takes in account contributions of all aberrations to the image quality of the optical system [12]. The RMS spot radius for three presented microlenses is given as a function of a field angle in Fig. 3.

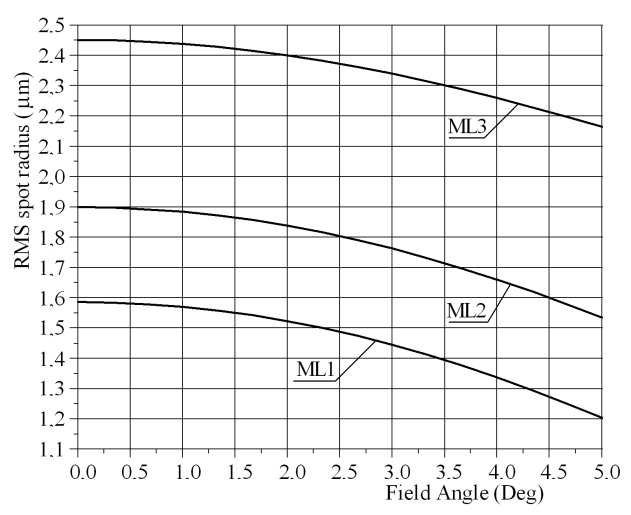

Fig. 3. RMS spot radius as a function of the clear aperture.

In order to make decision about the image quality of optical system the RMS spot radius is compared with the Airy disc radius which is calculated for a circular uniformly illuminated entrance pupil as [12]:

$$
r_{\mathrm{a}}=1.22 \lambda \mathrm{F} / \# \text {. }
$$


From Eq. (3) it can be seen that the Airy disc radius is function of $F$ number.

In Table III there are summarized values for the $F$ number, the Airy disc radius and the RMS spot radius for maximum field angle, for three microlenses.

TABLE III

The Airy disc radius and the RMS spot radius for three microlenses.

\begin{tabular}{l|c|c|c}
\hline \hline & ML1 & ML2 & ML3 \\
\hline$F$ number $(F / \#)[-]$ & 1.8 & 1.3 & 0.8 \\
Airy disc radius $[\mu \mathrm{m}]$ & 1.2 & 0.8 & 0.5 \\
RMS spot radius $[\mu \mathrm{m}]$ & 1.2 & 1.5 & 2.2
\end{tabular}

From data given in Table III and in Fig. 2 it can be seen that only microlens ML1 has near diffraction limited performance (the RMS spot radius is equal to the Airy disc radius). This is expected result because microlens ML1 has greatest effective focal length $(0.17 \mathrm{~mm})$ and smallest $F$ number $(f / 1.8)$. Microlenses ML2 and ML3 have the aberration limited performance. This is because microlenses ML2 and ML3 have similar RMS spot radius as the microlens ML1 and smaller Airy disk radius than the microlens ML1.

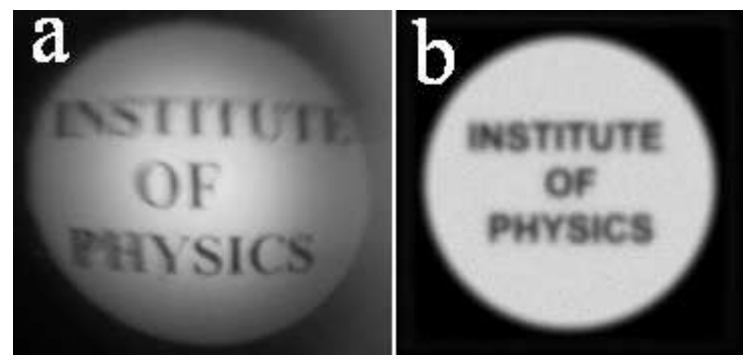

Fig. 4. (a) Image of "INSTITUTE OF PHYSICS" taken through the microscope and the microlens; (b) image of "INSTITUTE OF PHYSICS" calculated through the microlens by optical design software.

Figure 4 presents two photographs. The first is image of words "INSTITUTE OF PHYSICS" taken by digital camera through the classical microscope and the mi- crolens. The second is image of the same words calculated through the microlens by optical design program.

\section{Conclusions}

Three concave aspherical microlenses produced using the betanin sensitized gelatin and $532 \mathrm{~nm}$ laser irradiation are described. Presented microlenses have the large $F$ number and the moderate field angle. The longitudinal spherical aberration and the RMS spot radius are calculated. Only the microlens ML1 has the near diffraction limited performance. Microlenses ML2 and ML3 have similar aberrations to the microlens ML1 but have aberration limited performance because of large $F$ numbers.

\section{Acknowledgments}

This paper was written with the support of the Ministry of Science and Technological Development of the Republic of Serbia, under the project No. 141003.

\section{References}

[1] V. Wang, J. Fang, IEEE Sensors J. 7, 11 (2007).

[2] T. Tanaami, S. Otsuki, N. Tomosada, Y. Kosugi, M. Shimizu, H. Ishida, Appl. Opt. 41, 4704 (2005).

[3] L. Seifert, H.J. Tiziani, W. Osten, Opt. Commun. 245, 255 (2005).

[4] J. Kim, S. Nayak, L.A. Lyon, J. Am. Chem. Soc. 127, 9588 (2005).

[5] D.W. de Lima Monteiro, O. Akhzar-Mehr, P.M. Sarro, G. Vdovin, Opt. Exp. 11, 2244 (2003).

[6] R. Grunwald, S. Woggon, R. Ehlert, W. Reinecke, Pure Appl. Opt. 6, 663 (1997).

[7] B. Murić, D. Pantelić, D. Vasiljević, B. Panić, Opt. Mater. 30, 1217 (2008).

[8] B. Murić, D. Pantelić, D. Vasiljević, B. Panić, Appl. Opt. 46, 8527 (2007).

[9] B. Murić, D. Pantelić, D. Vasiljević, B. Panić, B. Jelenković, Appl. Opt. 48, 3854 (2009).

[10] D. Vasiljević, B. Murić, D. Pantelić, B. Panić, Acta Phys. Pol. A 112, 993 (2007).

[11] Sheet material US Patent 4481252, November 1984.

[12] V.N. Mahajan, Optical Imaging and Aberrations, SPIE Press, Bellingham, Washington 2001. 